\title{
The Use of Super Resolution in Robotic Assisted Minimally Invasive Surgery
}

\author{
Mirna Lerotic and Guang-Zhong Yang \\ Royal Society/Wolfson Foundation Medical Image Computing Laboratory, \\ Imperial College of Science, Technology and Medicine, London SW7 2BZ, UK \\ Mirna.Lerotic@imperal.ac.uk, g.z.yang@imperial.ac.uk
}

\begin{abstract}
In minimally invasive surgery, a small field-of-view is often required for achieving a large magnification factor during micro-scale tasks such as coronary anastomosis. Constant change of the orientation and focal length of the laparoscope camera, however, is cumbersome and can impose extra visual and cognitive load to the operating surgeon in realigning the visual pathways and anatomical landmarks. The purpose of this paper is to investigate the use of fixational movements for robotic assisted minimal invasive surgery such that the perceived resolution of the foveal field-of-view is greater than the intrinsic resolution of the laparoscope camera. The proposed technique is based on super resolution imaging using projection onto convex sets. Validation with both phantom and in vivo data from totally endoscopic coronary artery bypass surgery is provided.
\end{abstract}

\section{Introduction}

The use of computer assisted technology in surgical applications has risen dramatically in recent years, contributing to a range of new methods for training, education and diagnosis. In surgery, advances in medical image computing have permitted detailed preoperative planning and intra-operative surgical guidance. One of the most promising advances in surgical technology in recent years is the introduction of robotic assisted Minimally Invasive Surgery (MIS) [1]. It is increasingly being used to perform procedures that are otherwise prohibited by the confines of the operating environment. The technique offers a unique opportunity for deploying sophisticated surgical tools that can greatly enhance the manual dexterities of the operating surgeon. The future of robotic surgery is in the intelligent use of preoperative data for more complex procedures such as beating heart surgery where there is large-scale tissue deformation.

Whilst the benefit of patient recovery and surgical outcome is well established, the deployment of MIS is associated with the complexity of instrument controls, restricted vision and mobility, difficult hand-eye co-ordination, and the lack of tactile perception which require a high degree of operator dexterity [2]. For robotic assisted MIS, dexterity is enhanced with microprocessor controlled mechanical wrists, which allow motion scaling for reducing gross hand movements and performing micro-scale 
tasks that are otherwise not possible. The issue of resolving restricted vision, however, has so far achieved limited progress. This is largely due to the restriction on the number of trocar ports used during MIS and the small field of view (FOV) required for achieving a large magnification factor for performing micro-scale tasks such as coronary anastomosis. The consequence of restricted vision significantly affects the visual-spatial orientation of the surgeon and the awareness of the peripheral sites. During MIS, constant change of the orientation and focal length of the laparoscope camera is cumbersome and can impose extra visual and cognitive load to the operating surgeon in realigning the visual pathways and anatomical landmarks. In certain cases, this can also contribute to surgical errors as view rotation is known to affect surgical performances. The limited FOV has also made intraoperative surgical guidance difficult due to the paucity of unique surface features for aligning pre- or intra-operative 3D image data.

Recently, gaze contingent robotic control has attracted significant research interest due to its unique capability in coupling human visual perception with machine vision $[3,4]$. The research is based on the fact that in terms of visual acuity, human eyes do not have a uniform visual response, in fact, the best visual acuity is only within a visual angle of one to two degrees. This is called foveal vision, and for areas that we do not direct our eyes towards when observing a scene, we have to rely on a cruder representation of the objects offered by non-foveal vision, of which the visual acuity drops off dramatically from the centre of focus. The limited extent of the fovea demands the eyes being highly mobile and able to sweep across a large visual angle. As a result, the motion of the eyes is extremely varied in both the amplitude and frequency spectrum. During the periods of visual fixation, small eye movements continuously move the projection of the image on the retina. These fixational eye movements include small saccades, slow drifts, and physiological nystagmus. Existing research has shown that micro-motions, including both micro-saccades of the eyes [5] and subpixel (sub-sampling) movements of visual scene [6], can enhance visual resolution, although the mechanisms of the human visual system are unknown.

The purpose of this paper is to investigate the use of fixational movements to robotic assisted MIS such that the perceived resolution of the foveal FOV is greater than the intrinsic resolution of the laparoscope camera. In practice, this permits the use of a relatively large FOV for micro-surgical tasks such that the aforementioned drawbacks associated with the existing MIS setup can be avoided. With the proposed method, the position of the surgeon's gaze can be located by using binocular eye tracking and super resolution can then be used to provide a high resolution image to the foveal FOV. The proposed technique is based on super resolution imaging using projection onto convex sets (POCS) [7, 8] and validation with both phantom and in vivo data from totally endoscopic coronary artery bypass (TECAB) surgery is provided.

\section{Method}

Super resolution is a method used to get a high resolution image from a set of low resolution images that have sub-pixel shifts [6]. The additional information required 
to calculate the high resolution image is provided by different "views" given by subpixel shifts of low resolution images, and therefore provides a way of pushing the image resolution beyond the intrinsic limitation of the image sensors.

If one considers high resolution (HR) image $\mathbf{x}$ to be the true representation of the scene, then a low resolution (LR) image $\mathbf{y}_{i}$ is equal to

$$
\mathbf{y}_{i}=\mathbf{D M H}_{i} \mathbf{x}+\mathbf{v}_{i}
$$

where $\mathbf{D}$ is a down-sampling operator, $\mathbf{M}$ is a warp operator which accounts for translation, rotation and other transformations, $\mathbf{H}$ incorporates the point spread function (PSF) of the sensor such as motion blur, and $\mathbf{v}_{i}$ describes the additive noise. The recovery of the HR image $\mathbf{x}$ from a set of sub-pixel shifted LR images $\mathbf{y}_{i}$ is the goal of all super-resolution algorithms. Existing approaches to the problem include nonuniform interpolation (which consists of sub-pixel LR image registration, interpolation to the HR grid and image restoration), constrained least squares (CLS) [9], and maximum a posteriori (MAP) [10].

The super-resolution reconstruction algorithm used in this work is based on the method of projection onto convex sets (POCS) [7, 8]. The POCS method is an iterative method which incorporates prior knowledge by restricting the solution to the intersection of closed convex sets. In each iteration, a solution $\mathbf{x}^{n+1}$ is derived such that

$$
\mathbf{x}^{n+1}=P_{m} P_{m-1} \ldots P_{2} P_{1} \mathbf{x}^{n}
$$

where $P_{i}$ are projection operators which project the arbitrary solution $\mathrm{x}^{n}$ onto $\mathrm{m}$ closed convex sets. Each convex set represents a constraint on the solution such as amplitude, energy, or reference-image constraint [8]. In other words, the projection operators impose prior knowledge about the HR image on the solution.

Tekalp et al [7] introduced the following closed convex set constraint for each pixel of each low resolution frame $\mathbf{y}_{i}$

$$
C_{i}=\left\{\mathbf{x}_{k, l}:\left|\mathbf{r}_{i ; m, n}^{(\mathbf{x})}\right| \leq \delta_{0}\right\}
$$

where $\delta_{0}$ defines a bound that represents the statistical confidence in observed LR frames, and is usually estimated from the noise present in the LR images. The $\mathbf{r}_{i}^{(\mathrm{x})}$ in a sense represents the difference between the HR image $\mathrm{x}$ convolved with a PSF and the $i$-th LR image $\mathbf{y}_{i}$

$$
\mathbf{r}_{i ; m, n}^{(\mathbf{x})}=\mathbf{y}_{i ; m, n}-\sum_{k, l} \mathbf{x}_{k, l} \mathbf{h}_{i ; m, n ; k, l}
$$

where $\mathbf{h}_{i}$ is a PSF of the sensor for the $i$-th frame, given by the ratio of the area where LR pixel $(\mathrm{m}, \mathrm{n})$ and HR pixel $(\mathrm{k}, \mathrm{l})$ overlap divided by the total area of the LR pixel. The projection of a HR image $\mathbf{x}$ onto convex set $C_{i}$ defined in Eq. (3) is given by [7] 


$$
\mathbf{x}_{k, l}^{n+1}=\mathbf{x}_{k, l}^{n}+\left\{\begin{array}{c}
\frac{r_{i ; m, n}^{(x)} \mp \delta_{0}}{\sum_{o, p} \mathbf{h}_{i ; m, n ; o, p}^{2}} \mathbf{h}_{i ; m, n ; k, l} \text { if } \mathbf{r}_{i ; m, n}^{(x)}> \pm \delta_{0} \\
0 \quad \text { if }-\delta_{0} \leq \mathbf{r}_{i ; m, n}^{(x)} \leq \delta_{0}
\end{array}\right.
$$

In this study, the reconstruction of the HR images involves the use of the projection operator presented in Eq. (5) together with amplitude and energy constraints. For super-resolution imaging, accurate image registration is crucial to the subsequent reconstruction result, and for this reason the method proposed by Keren et al [11] was used.

In order to validate the proposed super-resolution framework for MIS, a Stäubli RX60 robotic arm was used to control the sub-pixel movement of the camera. The system has six degrees of freedom (DOF) and a repeatability accuracy of $\pm 0.02 \mathrm{~mm}$ at high speed and acceleration. A phantom heart model was created by using thixotropic silicone mould rubber and pre-vulcanised natural rubber latex with rubber mask grease paint to achieve a specular appearance and high visual fidelity. A video sequence was captured using a camera mounted on the described robotic arm. The camera was moved in a zig-zag pattern with the total movement spanning over few pixels. From the video sequence, 40 images were extracted and each image was separately down-sampled to one fourth of the original resolution, to ensure that the algorithm remains "blind" to individual frame shifts. To assess the in vivo applicability of the technique, a video sequence from a totally endoscopic coronary artery bypass surgery was used. The image shifts in this case were provided by the natural sub-pixel movement of the camera, present it the video.

\section{Results}

Fig. 1 shows the example images for the phantom heart experiment, where Fig. 1(a) shows a video snap-shot of the "true", actual scene (original image), Fig. 1(b) is the
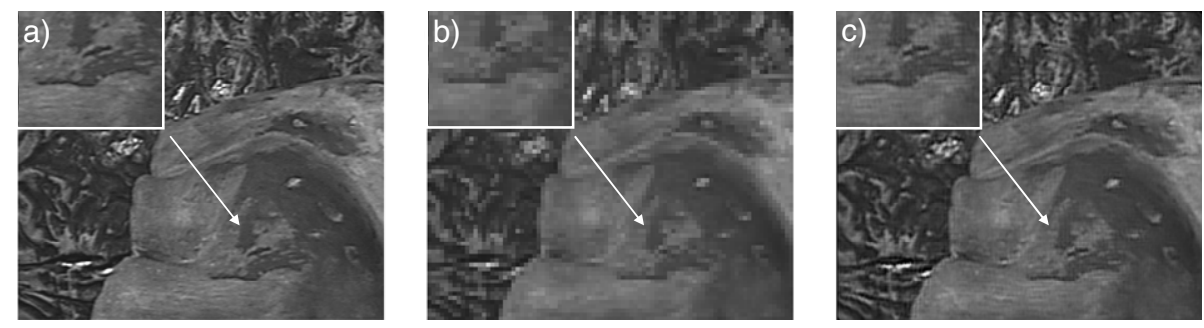

Fig. 1. (a) A video snap-shot of the actual scene (original image) and the corresponding LR images obtained by down-sampling (b). (c) The resulting image after the application of the described POCS super-resolution algorithm, where the insert demonstrates the high resolution details recovered by the proposed algorithm. 
corresponding LR image obtained by down-sampling, and Fig. 1(c) illustrates the result of the described POCS super-resolution algorithm as applied to the set of 40 registered LR images. The insert of each figure shows a magnified view of the FOV pointed by the arrow, demonstrating the high resolution-details that have been recovered by the proposed POCS algorithm.

To quantify the level of detail reconstructed in the HR image, an entropy measure [12] that represents the amount of the information present in the images is calculated. Larger entropy values are in general indicative of the high resolution details recovered. For the phantom study, the entropy value of the Original, LR, and POCS reconstructed HR images are 6.74, 6.57, and 6.72, respectively, which suggests the information recovered by the HR image is close to $99.7 \%$ based on the entropy measure. It should be noted, however, that this is a global statistical measure, and may not represent the actual visual fidelity recovered. Nevertheless, it does provide a quantitative index when combined with visual feature assessment.

In order to examine the convergence behavior of the algorithm, Fig. 2 (a) illustrates the RMS error in relation to the iteration steps. The algorithm in general has a rapid convergence rate, which makes it suitable for real-time implementations. In Fig. 2(bc), the power spectra of the LR and HR images are also provided, which demonstrate the amount of high-frequency details recovered.
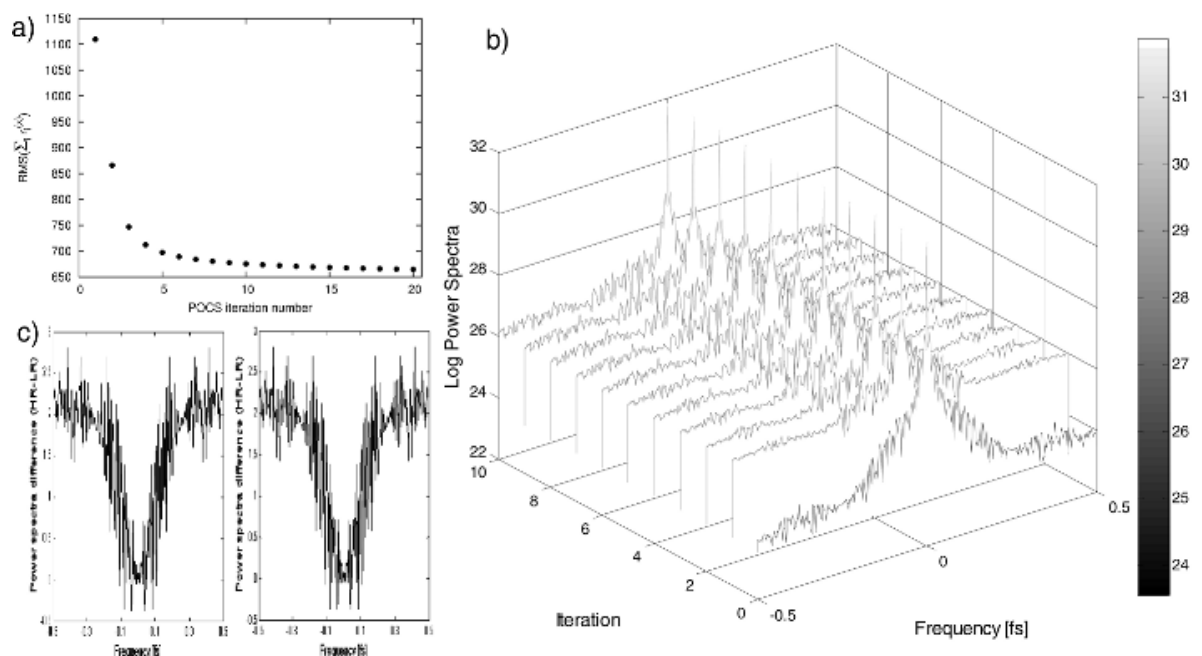

Fig. 2. (a) The convergence of the POCS algorithm as demonstrated by the RMS sum of $\mathbf{r}_{i}^{(\mathbf{x})}$ defined in Eq. (4). (b) Power spectra of the LR image (at zero position) and HR images for the first 10 iterations (where fs denotes the spatial sampling frequency). The plot shown in (c) shows a difference of power spectra (HR ( $10^{\text {th }}$ iteration)-LR). 
For in vivo data, Fig. 3 illustrates an FOV corresponding to the foveal region of the fixation and the high-resolution details recovered by the POCS algorithm, where the corresponding images before resolution enhancement are provided as a reference. The figure also indicates the position of the FOV in relation to the operating view. To provide a more accurate assessment of the level of details recovered, Fig. 4 illustrates the intensity profiles as marked in Fig. 3 for each of the images presented. The corresponding entropy measures for these images are summarized in Table 1.
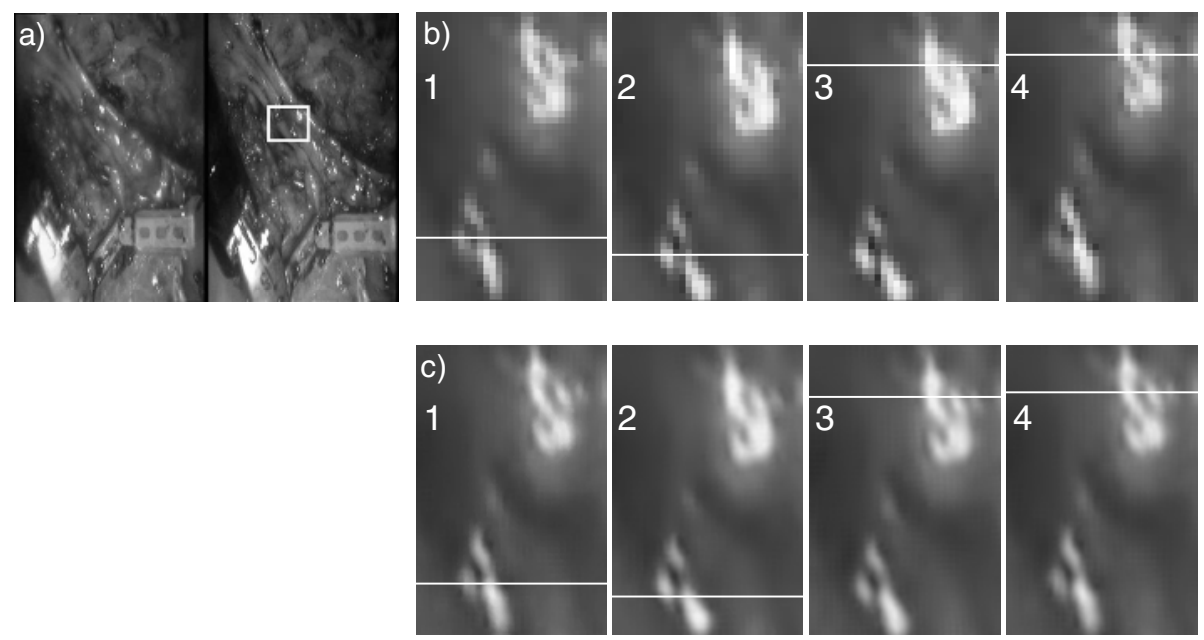

Fig. 3. (a) Image frames from a TECAB sequence; (b) the corresponding magnified time frames from the region marked by the white square in (a); (c) HR reconstructions calculated using the described POCS method. White lines on (b-c) indicate rows at which intensity profiles are shown in Fig. 4.

\section{Discussion and Conclusions}

In this paper, we have demonstrated the use of super resolution for robotic assisted minimally invasive surgery. This described method presents a novel approach to add an artificial zoom in MIS. The basic motivation of the technique is to investigate the use of fixational movements to robotic assisted MIS such that the perceived resolution of the foveal FOV is greater than the intrinsic resolution of the laparoscope camera. This will allow the use of a relatively large FOV for micro-surgical tasks such that super-resolution is applied to fixation points in response to real-time eye tracking. Experiments with both phantom data under controlled environment and time series from a TECAB surgery were used, and the results derived by using POCS reconstruction demonstrate the achieved improvement in spatial resolution. In combination with eye tracking, the described method can yield gaze contingent super-resolution foveal vision to the surgeon, thus minimizing the need to move the laparoscope camera during surgery. 

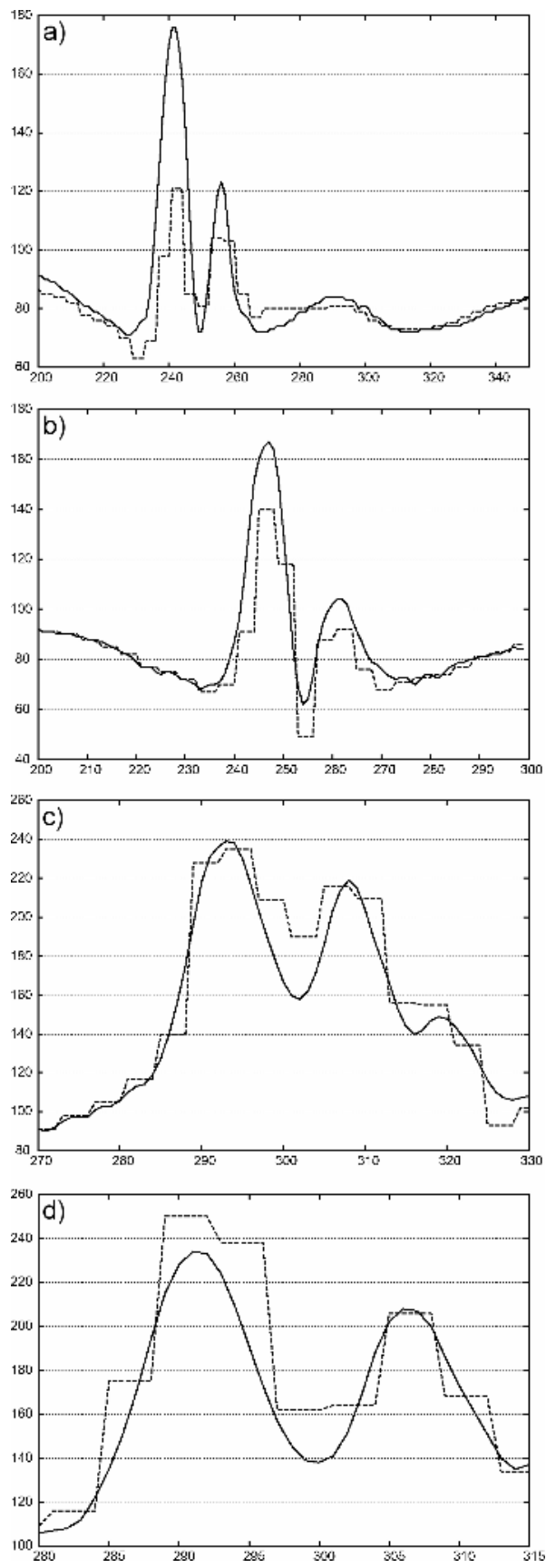

Fig. 4. (a)-(d) Pixel intensity profiles across image features on both HR (solid lines) and LR (dashed lines) images showing the improved resolution of the reconstructed image for the time series 1-4 shown in Fig. 3. Pixels used for line profiles are marked by white lines in Fig. 3(b-c). 
Table 1. Entropy values for the TECAB images shown in Figs. 3(b-c)

\begin{tabular}{cccc}
\hline Time series & LR image & HR image & Difference HR - LR \\
\hline 1 & 6.72 & 6.83 & 0.11 \\
\hline 2 & 6.78 & 6.82 & 0.04 \\
\hline 3 & 6.79 & 6.85 & 0.06 \\
\hline 4 & 6.79 & 6.84 & 0.05 \\
\hline
\end{tabular}

\section{Acknowledgements}

The authors would like to thank George P. Mylonas for the help in recording the video sequence of the phantom, and RCUK Basic Technology Programme for financial support.

\section{References}

1. Ballantyne, G.H., Robotic surgery, telerobotic surgery, telepresence, and telementoring. Surgical Endoscopy, 2002. 16(10): p. 1389-1402.

2. Tendick, F., et al., Sensing and Manipulation Problems in Endoscopic Surgery: Experiment, Analysis, and Observation. Presence, 1993. 2(1): p. 66-81.

3. Mylonas, G.P., A. Darzi, and G.-Z. Yang, Gaze Contingent Depth Recovery and Motion Stabilisation for Minimally Invasive Robotic Surgery. Lecture Notes in Computer Science. 2004. 311-319.

4. Mylonas, G.P., et al., Gaze-Contingent Soft Tissue Deformation Tracking for Minimally Invasive Robotic Surgery. Lecture Notes in Computer Science. 2005. 843-850.

5. Martinez-Conde, S., S.L. Macknik, and D.H. Hubel, The role of fixational eye movements in visual perception. Nature Reviews Neuroscience 2004. 5(3): p. 229-40.

6. Sung Cheol, P., P. Min Kyu, and K. Moon Gi, Super-resolution image reconstruction: a technical overview. Signal Processing Magazine, IEEE, 2003. 20(3): p. 21-36.

7. Tekalp, A.M., M.K. Ozkan, and M.I. Sezan. High-resolution image reconstruction from lower-resolution image sequences and space-varying image restoration. in IEEE International Conference on Acoustics, Speech, and Signal Processing, ICASSP-92. 1992, p. 169-172

8. Stark, H. and P. Oskoui, High-resolution image recovery from image-plane arrays, using convex projections. J. Opt. Soc. Am. A, 1989. 6(11): p. 1715-1726.

9. Hong, M.-C., M.G. Kang, and A.K. Katsaggelos. An Iterative Weighted Regularized Algorithm for Improving the Resolution of Video Sequences. in Proc. 1997 IEEE International Conf. on Image Processing. 1997, p. 474-477

10. Schultz, R.R. and R.L. Stevenson, Extraction of high-resolution frames from video sequences. Image Processing, IEEE Transactions on, 1996. 5(6): p. 996-1011.

11. Keren, D., S. Peleg, and R. Brada. Image sequence enhancement using sub-pixel displacements. in Proceedings CVPR '88., Computer Society Conference on Computer Vision and Pattern Recognition. 1988. Ann Arbor, MI, USA, p. 742-746

12. Gonzalez, R.C. and R.E. Woods, Digital Image Processing. 2002, New Jersey: PrenticeHall, Inc. 